International Journal of Pure and Applied Mathematics

Volume 112 No. 2 2017, 239-252

ISSN: 1311-8080 (printed version); ISSN: 1314-3395 (on-line version)

url: http://www.ijpam.eu

doi: 10.12732 /ijpam.v112i2.2

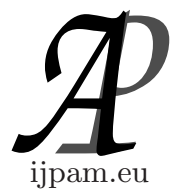

\title{
ON THE HYPER-ZAGREB INDEX OF SOME OPERATIONS ON GRAPHS
}

\author{
V. Anandkumar ${ }^{1 \S}$, Radha Rajamani Iyer $^{2}$ \\ ${ }^{1}$ Department of Mathematics \\ EASA College of Engineering and Technology \\ Coimbatore, INDIA \\ ${ }^{2}$ Department of Mathematics \\ Amrita School of Engineering \\ Coimbatore, Amrita Vishwa Vidyapeetham \\ Amrita University, INDIA
}

\begin{abstract}
Let $\mathrm{G}$ be any (molecular) graph. The hyper-Zagreb index of a graph was introduced and studied by G.H Shirdel and H.Rezapour[22]. In this paper some exact expressions for the hyper-Zagreb index of four graph operations(F-sums) of a graph are established.
\end{abstract}

AMS Subject Classification: 05C76, 05C90

Key Words: Zagreb index, F-sums, topological index, hyper-Zagreb index

\section{Introduction}

In general, a topological index, also known as a graph-theoretic index, is a numerical invariant of a chemical graph (Plavšić et.al. 1993) [20]. There are two major types of topological indices, namley the distance based and the degree based. The very first paper studied in this aspect was the Wiener index of a graph by using distance between pair of vertices in the graph by the famous chemist Herold Wiener while he was trying to find the boiling point of paraffin [22] by considering the structural property of molecular graphs. Another topo-

Received: $\quad$ October 6, 2016

Revised: $\quad$ November 24, 2016

Published: January 27, 2017

${ }^{\S}$ Correspondence author (c) 2017 Academic Publications, Ltd. url: www.acadpubl.eu 
logical index is the Zagreb indices, which dates back to 30 years, see $[9,19]$. The first and second Zagreb indices of a graph are the most studied degree based topological indices introduced by Gutman and Trinajstic(1972) used to analyze the structure-dependency of the total $\pi$-electron energy $(\varepsilon)$, see [10]. For more historical background of Zagreb indices we refer to [2,3,5,7,14-18,23].

G.H Shirdel and H.Rezapour introduced the hyper-Zagreb index of a graph in 2013 and studied for some graph operations. Recently B.Basavanagoud and S.Patil published a paper on hyper-Zagreb index[2] and rectified some errors of [22]. Wei gao et.al. studied hyper-Zagreb for cartesian product of some special graphs and applied these results in some chemical graphs [21]. Eliasi and Taeri [7] introduced four new operations of F-sums and obtained the expression for the Wiener index $W\left(G_{1}+{ }_{F} G_{2}\right)$ of $G_{1}+{ }_{F} G_{2}$ in terms of $W\left(F\left(G_{1}\right)\right)$ and $W\left(G_{2}\right)$. Recently in[11], first and second Zagreb indices for the F-sums of graphs were presented. In this paper we obtain the explicit formulae for hyper-Zagreb index of a graph for four operations in F-sums. For the computational techniques we refer $[8,11,12,13]$.

\section{Definition and Preliminaries}

Let $\mathrm{G}$ be a simple connected undirected graph with a vertex set $V(G)$ and edge set $E(G)$. A topological representation of a molecule is known as molecular graph. A molecular graph is a collection of vertices representing atoms of molecule and edges representing the covalent bonds between the atoms. Topological indices can be used directly as the numerical values in biological parameters of molecules in quantitative structure-property relationship (QSPR) and quantitative structure-activity relationship (QSAR) [6]. For any vertex $v \epsilon V(G)$, $d_{G}(v)$ denotes the degree of a vertex $v$ in $G$. The first and second Zagreb indices of a graph $G$ are denoted by $M_{1}(G)$ and $M_{2}(G)$ and they are defined as,

$$
M_{1}(G)=\sum_{v \in V(G)} d(v)^{2} \text { and } M_{2}(G)=\sum_{u v \in E(G)} d(u) d(v) .
$$

The hyper-Zagreb index of a graph $G$ is denoted by $H M(G)$ and defined as

$$
H M(G)=\sum_{u v \in E(G)}[d(u)+d(v)]^{2} .
$$

Cartesian product of two connected graphs $G_{1}$ and $G_{2}$, denoted by $G_{1} \times G_{2}$, is a graph such that the set of vertices is $V\left(G_{1}\right) \times V\left(G_{2}\right)$ and two vertices $u=\left(u_{1}, v_{1}\right)$ and $v=\left(u_{2}, v_{2}\right)$ of $G_{1} \times G_{2}$ are adjacent if and only if $\left[u_{1}=u_{2}\right.$ and $v_{1}$ is adjacent with $v_{2}$ in $\left.G_{2}\right]$ or $\left[v_{1}=v_{2}\right.$ and $u_{1}$ is adjacent with $u_{2}$ in $G_{1}$ ]. 


\subsection{F-Sums}

For any simple finite connected graph $G$,

1. $S(G)$ is the sub-division graph of $G$.

2. $R(G)$ is semi-total(point) graph.

3. $Q(G)$ is semi-total(line) graph.

4. $T(G)$ is the total graph of $G$.

Suppose that $G_{1}$ and $G_{2}$ are two connected graphs. Based on these above operations, four new operations on these graphs are as defined below:

Let $F \in S, R, Q, T$. The $F$-sum of $G_{1}$ and $G_{2}$, denoted by $G_{1}+{ }_{F} G_{2}$, is defined as $F\left(G_{1}\right) \times G_{2}-E^{*}$, where $E^{*}=\left\{\left(u, v_{1}\right)\left(u, v_{2}\right) \in E\left(F\left(G_{1}\right) \times G_{2}\right)\right.$ : $\left.u \in V\left(F\left(G_{1}\right)-V(G), v_{1} v_{2} \in E\left(G_{2}\right)\right)\right\}$, i.e., $G_{1}+{ }_{F} G_{2}$ is a graph with the set of vertices $V\left(G_{1}+{ }_{F} G_{2}\right)=\left(V\left(G_{1}\right) \cup E\left(G_{1}\right)\right) \times V\left(G_{2}\right)$ and two vertices $\left(u_{1}, u_{2}\right)$ and $\left(v_{1}, v_{2}\right)$ of $G_{1}+{ }_{F} G_{2}$ are adjacent if and only if $\left[u_{1}=v_{1} \in V\left(G_{1}\right)\right.$ and $\left.u_{2} v_{2} \in E\left(G_{2}\right)\right]$ or $\left[u_{2}=v_{2} \in V\left(G_{2}\right)\right.$ and $\left.u_{1} v_{1} \in E\left(F\left(G_{1}\right)\right)\right]$. The Figure 1 and Figure 2 depicts the graphs of the four operations $G_{1}+{ }_{S} G_{2}, G_{1}+{ }_{R} G_{2}, G_{1}+{ }_{Q} G_{2}$ and $G_{1}+{ }_{T} G_{2}$ clearly.

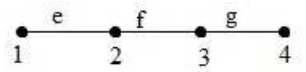

$\mathrm{G}=\mathrm{P}_{4}$

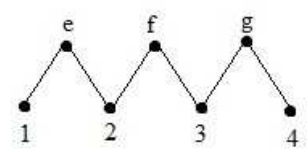

$\mathrm{S}\left(\mathrm{P}_{4}\right)$

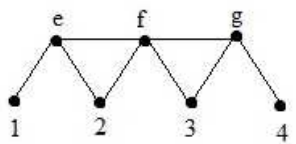

$\mathrm{Q}\left(\mathrm{P}_{4}\right)$

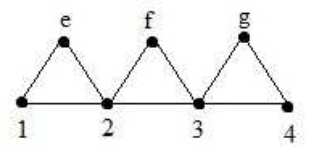

$\mathrm{R}\left(\mathrm{P}_{4}\right)$

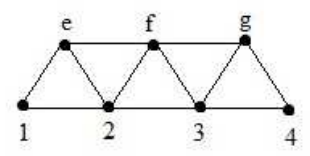

$\mathrm{T}\left(\mathrm{P}_{4}\right)$

Fig.l. $\mathrm{P}_{4}, \mathrm{~S}\left(\mathrm{P}_{4}\right), \mathrm{R}\left(\mathrm{P}_{4}\right), \mathrm{Q}\left(\mathrm{P}_{4}\right)$ and $\mathrm{T}\left(\mathrm{P}_{4}\right)$. 

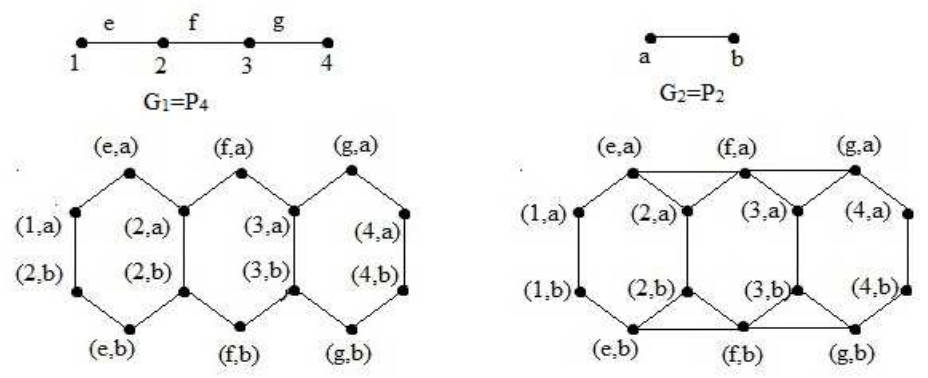

$\mathrm{G}_{1}+\mathrm{s} \mathrm{G}_{2}$

$\mathrm{G}_{1}+\mathrm{Q}_{\mathrm{Q}}$
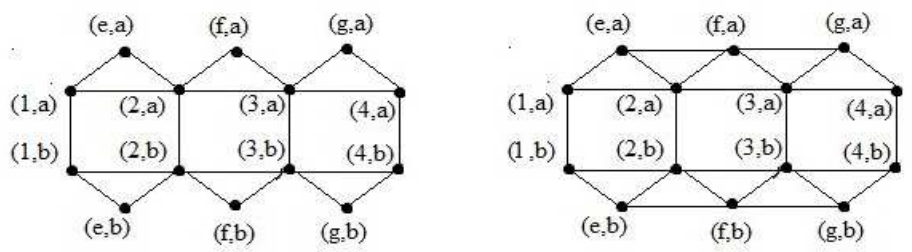

$\mathrm{G}_{1}+\mathrm{R} \mathrm{G}_{2}$

$\mathrm{G}_{1}+\mathrm{T}_{2}$

Fig.2. $\mathrm{G}_{1}, \mathrm{G}_{2}, \mathrm{G}_{1}+\mathrm{s} \mathrm{G}_{2}, \mathrm{G}_{1}+\mathrm{R} \mathrm{G}_{2}, \mathrm{G}_{1}+{ }_{\mathrm{Q}} \mathrm{G}_{2}, \mathrm{G}_{1}+{ }_{\mathrm{I}} \mathrm{G}_{2}$

\section{Main Results}

In this section we obtain the results of hyper-Zagreb index of graphs for four operations of F-sums.

Theorem 3.1. Let $G_{1}$ and $G_{2}$ be two connected graphs. Then

$$
\begin{aligned}
H M\left(G_{1}+{ }_{S} G_{2}\right)=4 e_{2} M_{1}\left(G_{1}\right)+n_{1} H M\left(G_{2}\right) & +10 e_{1} M_{1}\left(G_{2}\right) \\
& +n_{2} H M\left(S\left(G_{1}\right)\right)+4 e_{2} M_{1}\left(S\left(G_{1}\right)\right) .
\end{aligned}
$$

Proof. We have

$$
H M\left(G_{1}+{ }_{S} G_{2}\right)=\sum_{u, v \in E\left(G_{1}\right)}(d(u)+d(v))^{2}
$$


ON THE HYPER-ZAGREB INDEX...

$$
\begin{aligned}
& =\sum_{\left(u_{1}, v_{1}\right)\left(u_{2}, v_{2}\right) \in E\left(G_{1}+S_{S} G_{2}\right)}\left[d\left(u_{1}, v_{1}\right)+d\left(u_{2}, v_{2}\right)\right]^{2} \\
& =\sum_{u \in V\left(G_{1}\right)} \sum_{v_{1}, v_{2} \in E\left(G_{1}\right)}\left[d\left(u, v_{1}\right)+d\left(u, v_{2}\right)\right]^{2} \\
& \quad+\sum_{v \in V\left(G_{2}\right)} \sum_{u_{1}, u_{2} \in E\left(S\left(G_{1}\right)\right)}\left[d\left(u_{1}, v\right)+d\left(u_{2}, v\right)\right]^{2} \\
& =(1)+(2),
\end{aligned}
$$

$$
\begin{aligned}
(1) \Rightarrow & \sum_{u \in V\left(G_{1}\right)} \sum_{v_{1}, v_{2} \in E\left(G_{2}\right)}\left[\left(d_{G_{1}}(u)+d_{G_{2}}\left(v_{1}\right)\right)+d_{G_{1}}(u)+d_{G_{2}}\left(v_{2}\right)\right]^{2} \\
= & \sum_{u \in V\left(G_{1}\right)} \sum_{v_{1}, v_{2} \in E\left(G_{2}\right)}\left[2 d_{G_{1}}(u)+d_{G_{2}}\left(v_{1}\right)+d_{G_{2}}\left(v_{2}\right)\right]^{2} \\
= & \sum_{u \in V\left(G_{1}\right)}\left[4\left(d_{G_{1}}(u)\right)^{2}+2 d_{G_{1}}(u) d_{G_{2}}\left(v_{1}\right)\right. \\
& +2 d_{G_{1}}(u) d_{G_{2}}\left(v_{2}\right)+\left(d_{G_{1}}\left(v_{1}\right)\right)^{2}+2 d_{G_{1}}(u) d_{G_{2}}\left(v_{1}\right) \\
& \left.+d_{G_{2}}\left(v_{1}\right) d_{G_{2}}\left(v_{2}\right)+\left(d_{G_{2}}\left(v_{2}\right)\right)^{2}+2 d_{G_{1}}(u) d_{G_{2}}\left(v_{2}\right)+d_{G_{2}}\left(v_{1}\right) d_{G_{2}}\left(v_{2}\right)\right] \\
= & \sum_{u \in V\left(G_{1}\right)}\left[4\left(d_{G_{1}}(u)\right)^{2}+d_{G_{2}} \in E\left(v_{1}\right)^{2}\right. \\
& \left.+d_{G_{2}}\left(v_{2}\right)^{2}+4 d_{G_{1}}(u) d_{G_{2}}\left(v_{1}\right)+4 d_{G_{1}}(u) d_{G_{2}}\left(v_{2}\right)+2 d_{G_{2}}\left(v_{1}\right) d_{G_{2}}\left(v_{2}\right)\right] \\
= & 4 e_{2} M_{1}\left(G_{1}\right)+n_{1} H M\left(G_{2}\right)+\sum_{u \in V\left(G_{1}\right)} \sum_{v_{1} v_{2} \in E\left(G_{2}\right)} 4 d_{G_{1}}(u)\left[d_{G_{2}}\left(v_{1}\right)+d_{G_{2}}\left(v_{2}\right)\right] \\
= & \left.4 e_{2} M_{1}\left(G_{1}\right)+n_{1} H M\left(G_{2}\right)+8 e_{1} M_{1}\left(G_{2}\right)\right),
\end{aligned}
$$

$$
\begin{aligned}
&(2) \Rightarrow \sum_{v \in V\left(G_{2}\right)} \sum_{\substack{u_{1}, u_{2} \in E\left(S\left(G_{1}\right)\right) \\
u_{2} \in V\left(S\left(G_{1}\right)\right)-V\left(G_{1}\right)}}\left[d_{S\left(G_{1}\right)}\left(u_{1}\right)+d_{G_{2}}(v)+d_{S\left(G_{1}\right)}\left(u_{2}\right)\right]^{2} \\
&=\sum_{v \in V\left(G_{2}\right)} \sum_{\substack{u_{1}, u_{2} \in E\left(S\left(G_{1}\right)\right) \\
u_{2} \in V\left(S\left(G_{1}\right)\right)-V\left(G_{1}\right)}}\left[\left(d_{S\left(G_{1}\right)}\left(u_{1}\right)\right)^{2}+2 d_{S\left(G_{1}\right)}\left(u_{1}\right) d_{G_{2}}(v)\right. \\
&\left.+2 d_{S\left(G_{1}\right)}\left(u_{1}\right) d_{S\left(G_{1}\right)}\left(u_{2}\right)+\left(d_{G_{2}}(v)\right)^{2}+2 d_{G_{2}}(v) d_{S\left(G_{1}\right)}\left(u_{2}\right)+d_{S\left(G_{1}\right)}\left(u_{2}\right)^{2}\right] \\
&= n_{2} H M\left(G_{1}\right)+\sum_{v \in V(G)}\left[2 ( d _ { G _ { 2 } } ( v ) ) \left[d_{S\left(G_{1}\right)}\left(u_{1}\right)\right.\right.
\end{aligned}
$$




$$
\begin{aligned}
& \left.\left.+d_{S\left(G_{1}\right)}\left(u_{2}\right)\right]+\left(d_{G_{2}}(v)\right)^{2}\right]+2.2 e_{2} M_{1}\left(S\left(G_{1}\right)\right)+M_{1}\left(G_{2}\right) 2 e_{2} \\
= & n_{2} H M\left(S\left(G_{1}\right)\right)+4 e_{2} M_{1}\left(S\left(G_{1}\right)\right)+2 e_{2} M_{1}\left(G_{2}\right) .
\end{aligned}
$$

Using (1) and (2), we receive

$$
\begin{aligned}
H M\left(G_{1}+{ }_{S} G_{2}\right)=4 e_{2} M_{1}\left(G_{1}\right)+n_{1} H M & \left(G_{2}\right)+10 e_{1} M_{1}\left(G_{2}\right) \\
& +n_{2} H M\left(S\left(G_{1}\right)\right)+4 e_{2} M_{1}\left(S\left(G_{1}\right)\right) .
\end{aligned}
$$

Theorem 3.2. Let $G_{1}$ and $G_{2}$ be two connected graphs. Then

$$
\begin{aligned}
H M\left(G_{1}+{ }_{R} G_{2}\right)=8 n_{2}\left[F\left(G_{1}\right)\right. & \left.+M_{1}\left(G_{1}\right)+M_{2}\left(G_{1}\right)\right]+2 e_{1}\left(11 M_{1}\left(G_{2}\right)\right. \\
& \left.+4 n_{2}\right)+8 e_{2}\left(5 M_{1}\left(G_{1}\right)+2 e_{1}\right)+n_{1} H M\left(G_{2}\right) .
\end{aligned}
$$

Proof. We receive

$$
\begin{aligned}
H M\left(G_{1}+{ }_{R} G_{2}\right)= & \sum_{\left(u_{1}, v_{1}\right),\left(u_{2}, v_{2}\right) \in E\left(G_{1}+{ }_{R} G_{2}\right)}\left[d\left(u_{1}, v_{1}\right)+d\left(u_{2}, v_{2}\right)\right]^{2} \\
= & \sum_{u \in V\left(G_{1}\right)} \sum_{v_{1} v_{2} \in E\left(G_{2}\right)}\left[d\left(u, v_{1}\right)+d\left(u, v_{2}\right)\right]^{2} \\
& +\sum_{v \in V\left(G_{2}\right)} \sum_{u_{1}, u_{2} \in E\left(R\left(G_{1}\right)\right)}\left[d\left(u_{1}, v\right)+d\left(u_{2}, v\right)\right]^{2} \\
= & (1)+(2) ;
\end{aligned}
$$

$$
\begin{aligned}
(1) \Rightarrow & \sum_{u \in V\left(G_{1}\right)} \sum_{v_{1} v_{2} \in E\left(G_{2}\right)}\left[2 d_{R\left(G_{1}\right)}(u)+d_{G_{2}}\left(v_{1}\right)+d_{G_{2}}\left(v_{2}\right)\right]^{2} \\
= & \sum_{u \in V\left(G_{1}\right)} \sum_{v_{1} v_{2} \in E\left(G_{2}\right)}\left[4 d_{R\left(G_{1}\right)}(u)^{2}+2 d_{R\left(G_{1}\right)}(u) d_{G_{2}}\left(v_{1}\right)\right. \\
& +2 d_{R\left(G_{1}\right)}(u) d_{G_{2}}\left(v_{2}\right)+d_{G_{2}}\left(v_{1}\right)^{2}+2 d_{R\left(G_{1}\right)}(u) d_{G_{2}}\left(v_{1}\right)+d_{G_{2}}\left(v_{1}\right) d_{G_{2}}\left(v_{2}\right) \\
& \left.+d_{G_{2}}\left(v_{2}\right)^{2}+2 d_{R\left(G_{1}\right)}(u) d_{G_{2}}\left(v_{2}\right)+d_{G_{2}}\left(v_{1}\right) d_{G_{2}}\left(v_{2}\right)\right] \\
= & \sum_{u \in V\left(G_{1}\right)} \sum_{v_{1} v_{2} \in E\left(G_{2}\right)}\left[16 d_{G_{1}}(u)^{2}+8 d_{G_{1}}(u) d_{G_{2}}\left(v_{1}\right)+d_{G_{2}}\left(v_{1}\right)^{2}\right. \\
& \left.+8 d_{G_{1}}(u) d_{G_{2}}\left(v_{2}\right)+d_{G_{2}}\left(v_{2}\right)^{2}+2 d_{G_{2}}\left(v_{1}\right) d_{G_{2}}\left(v_{2}\right)\right] \\
= & 16 e_{2} M_{1}\left(G_{1}\right)+n_{1} F\left(G_{2}\right)+16 e_{1} M_{1}\left(G_{2}\right)+2 n_{1} M_{2}\left(G_{2}\right) \text { (or) }
\end{aligned}
$$




$$
=16 e_{2} M_{1}\left(G_{1}\right)+n_{1} H M\left(G_{2}\right)+16 e_{1} M_{1}\left(G_{2}\right),
$$

$$
\begin{aligned}
& (2) \Rightarrow \sum_{v \in V\left(G_{2}\right)} \sum_{\substack{u_{1}, u_{2} \in E\left(R\left(G_{1}\right)\right) \\
u_{1}, u_{2} \in V(G)}}\left[d\left(u_{1}, v\right)+d\left(u_{2}, v\right)\right]^{2} \\
& =\sum_{v \in V\left(G_{2}\right)} \sum_{\substack{u_{1}, u_{2} \in E\left(R\left(G_{1}\right)\right) \\
u_{1}, u_{2} \in V\left(G_{1}\right)}}\left[d^{2}\left(u_{1}, v\right)+d^{2}\left(u_{2}, v\right)\right] \\
& +\sum_{v \in V\left(G_{2}\right)} \sum_{u_{1}, u_{2} \in E\left(R\left(G_{1}\right)\right)}\left[d^{2}\left(u_{1}, v\right)+d^{2}\left(u_{2}, v\right)\right] \\
& u_{1} \in V\left(G_{1}\right), u_{2} \in V\left(R\left(G_{1}\right)\right)-V\left(G_{1}\right) \\
& =(2 a)+(2 b) \text {; }
\end{aligned}
$$

$$
\begin{aligned}
(2 a) \Rightarrow \sum_{v \in V\left(G_{2}\right)} \sum_{\substack{u_{1}, u_{2} \in E\left(R\left(G_{1}\right)\right) \\
u_{1}, u_{2} \in V\left(G_{1}\right)}}\left[d\left(u_{1}, v\right)+d\left(u_{2}, v\right)\right]^{2} .
\end{aligned}
$$

Since here we consider edges of $G$ only, then

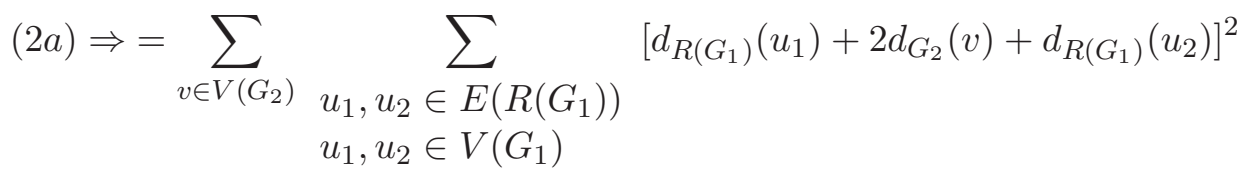

$$
\begin{aligned}
& =\sum_{v \in V\left(G_{2}\right)} \sum_{u_{1}, u_{2} \in V\left(G_{1}\right)}\left[4 d_{G_{1}}\left(u_{1}\right)^{2}+4 d_{G_{2}}(v)^{2}+4 d_{G_{1}}\left(u_{2}\right)^{2}\right. \\
& \left.+8 d_{G_{1}}\left(u_{1}\right) d_{G_{2}}(v)+8 d_{G_{2}}(v) d_{G_{1}}\left(u_{2}\right)+8 d_{G_{1}}\left(u_{1}\right) d_{G_{1}}\left(u_{2}\right)\right] \\
& =4 e_{1} M_{1}\left(G_{2}\right)+4 n_{2} H M\left(G_{1}\right)+16 e_{2} M_{1}\left(G_{1}\right) \text {, } \\
& (2 b) \Rightarrow \sum_{v \in V\left(G_{2}\right)} \sum_{u_{1}, u_{2} \in E\left(R\left(G_{1}\right)\right)}\left[d_{R\left(G_{1}\right)}\left(u_{1}\right)+d_{G_{2}}(v)+d_{R\left(G_{1}\right)}\left(u_{2}\right)\right]^{2} \\
& u_{1} \in V\left(G_{1}\right) \\
& u_{2} \in V\left(R\left(G_{1}\right)\right)-V\left(G_{1}\right)
\end{aligned}
$$

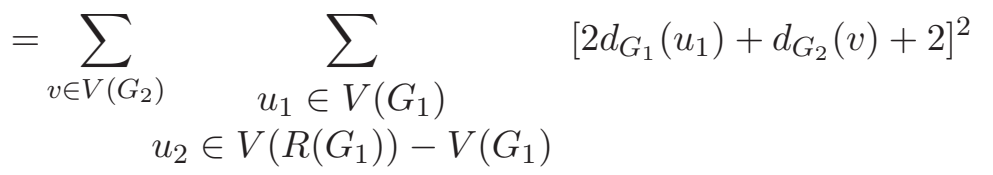




$$
\begin{aligned}
&= \sum_{v \in V\left(G_{2}\right)} \sum_{u_{1} \in V\left(G_{1}\right)}\left[4 d_{G_{1}}\left(u_{1}\right)^{2}+d_{G_{2}}(v)^{2}\right. \\
& u_{2} \in V\left(R\left(G_{1}\right)\right)-V\left(G_{1}\right) \\
&\left.+4+4 d_{G_{1}}\left(u_{1}\right) d_{G_{2}}(v)+4 d_{G_{2}}(v)+8 d_{G_{1}}\left(u_{1}\right)\right] \\
&= 4 n_{2} F\left(G_{1}\right)+2 e_{1} M_{1}\left(G_{2}\right)+4.2 e_{2} M_{1}\left(G_{1}\right)+4 n_{2} .2 e_{1} \\
& \therefore(2 b)= 4 n_{2} F\left(G_{1}\right)+2 e_{1} M_{1}\left(G_{2}\right)+8 n_{2} e_{1}+8 e_{2} M_{1}\left(G_{1}\right) \\
&+8 n_{2} M_{1}\left(G_{1}\right)+16 e_{1} e_{2} \\
&(2)=(2 a)+(2 b)=8 n_{2}\left[F\left(G_{1}\right)+M_{1}\left(G_{1}\right)+M_{2}\left(G_{1}\right)\right] \\
&+2 e_{1}\left(3 M_{1}\left(G_{2}\right)+4 n_{2}\right)+8 e_{2}\left(3 M_{1}\left(G_{1}\right)+2 e_{1}\right) .
\end{aligned}
$$

Hence, (1) and (2) gives

$$
\begin{aligned}
& H M\left(G_{1}+{ }_{R} G_{2}\right)=8 n_{2}\left[F\left(G_{1}\right)+M_{1}\left(G_{1}\right)+M_{2}\left(G_{1}\right)\right] \\
& +2 e_{1}\left(11 M_{1}\left(G_{2}\right)+4 n_{2}\right)+8 e_{2}\left(5 M_{1}\left(G_{1}\right)+2 e_{1}\right)+n_{1} H M\left(G_{2}\right) .
\end{aligned}
$$

Theorem 3.3. Let $G_{1}$ and $G_{2}$ be connected graphs. Then

$$
\begin{gathered}
H M\left(G_{1}+{ }_{Q} G_{2}\right)=16 e_{2} M_{1}\left(G_{1}\right)+n_{1} F\left(G_{2}\right)+10 e_{1} M_{1}\left(G_{2}\right)+2 M_{2}\left(G_{2}\right)+M_{2}\left(G_{1}\right) \\
+\left(n_{2}+2\right) F\left(G_{1}\right)+4\left(M_{1}\left(G_{1}\right)\right)^{2}+n_{2}\left[H M\left(L\left(G_{1}\right)\right)+8 M_{1}\left(L\left(G_{1}\right)\right)+16\right] .
\end{gathered}
$$

Proof. We have

$$
\begin{aligned}
H M\left(G_{1}+{ }_{Q} G_{2}\right)= & \sum_{\left(u_{1}, v_{1}\right)\left(u_{2}, v_{2}\right) \in E\left(G_{1}+{ }_{Q} G_{2}\right)}\left[d\left(u_{1}, v_{1}\right)+d\left(u_{2}, v_{2}\right)\right]^{2} \\
= & \sum_{u \in V\left(G_{1}\right)} \sum_{v_{1} v_{2} \in E\left(G_{2}\right)}\left[d\left(u, v_{1}\right)+d\left(u, v_{2}\right)\right]^{2} \\
& +\sum_{v \in V\left(G_{2}\right)} \sum_{u_{1} u_{2} \in E\left(Q\left(G_{1}\right)\right)}\left[d\left(u_{1}, v\right)+d\left(u_{2}, v\right)\right]^{2} \\
= & (1)+(2) .
\end{aligned}
$$

Moreover

$$
\begin{aligned}
(1) & \Rightarrow \sum_{u \in V\left(G_{1}\right)} \sum_{v_{1}}\left[2 d_{Q\left(G_{1}\right)}(u)+d_{G_{2}}\left(v_{1}\right)+d_{G_{2}}\left(v_{2}\right)\right]^{2} \\
& =\sum_{u \in V\left(G_{1}\right)} \sum_{v_{1}}\left[4 \cdot d_{Q\left(G_{1}\right)}(u)^{2}+d_{G_{2}}\left(v_{1}\right)^{2}+d_{G_{2}}\left(v_{2}\right)^{2}+4 . d_{Q\left(G_{1}\right)}(u) d_{G_{2}}\left(v_{1}\right)\right.
\end{aligned}
$$




$$
\begin{aligned}
& \left.+4 . d_{Q\left(G_{1}\right)}(u) d_{G_{2}}\left(v_{2}\right)+2 . d_{G_{2}}\left(v_{1}\right) d_{G_{2}}\left(v_{2}\right)\right] \\
= & \sum_{u \in V\left(G_{1}\right)} \sum_{v_{1} v_{2} \in E\left(G_{2}\right)}\left[4 . d_{G_{1}}(u)^{2}\right. \\
& \left.+d_{G_{2}}\left(v_{1}\right)^{2}+d_{G_{2}}\left(v_{2}\right)^{2}+4 . d_{G_{1}}(u)\left[d_{G_{2}}\left(v_{1}\right)+d_{G_{2}}\left(v_{2}\right)+2 . d_{G_{2}}\left(v_{1}\right) d_{G_{2}}\left(v_{2}\right)\right]\right] \\
= & 4 e_{2} M_{1}\left(G_{1}\right)+n_{1} F\left(G_{2}\right)+8 e_{1} M_{1}\left(G_{2}\right)+2 n_{1} M_{2}\left(G_{2}\right)
\end{aligned}
$$

and

$$
\begin{aligned}
(2) \Rightarrow \sum_{v \in V\left(G_{2}\right)} \sum_{\substack{u_{1} u_{2} \in E\left(Q\left(G_{1}\right)\right) \\
u_{1} \in V\left(G_{1}\right), u_{2} \in V\left(Q\left(G_{1}\right)\right)-V(G)}}\left[d\left(u_{1}, v\right)+d\left(u_{2}, v\right)\right]^{2} \\
\quad+\sum_{v \in V\left(G_{2}\right)} \sum_{\substack{u_{1} u_{2} \in E\left(Q\left(G_{1}\right)\right) \\
u_{1}, u_{2} \in V\left(Q\left(G_{1}\right)\right)-V(G)}}\left[d\left(u_{2}, v\right)\right]^{2} \\
=(2 a)+(2 b),
\end{aligned}
$$

where

$$
\begin{aligned}
& (2 a) \Rightarrow \sum_{v \in V\left(G_{2}\right)} \sum_{u_{1} u_{2} \in E\left(Q\left(G_{1}\right)\right)}\left[\left(d_{Q\left(G_{1}\right)}\left(u_{1}\right)\right.\right. \\
& u_{1} \in V\left(G_{1}\right), u_{2} \in V\left(Q\left(G_{1}\right)\right)-V\left(G_{1}\right) \\
& \left.\left.+d_{G_{2}}(v)\right)+d_{Q\left(G_{1}\right)}\left(u_{2}\right)\right]^{2} \\
& =\sum_{v \in V\left(G_{2}\right)} \sum_{u_{1} u_{2} \in E\left(Q\left(G_{1}\right)\right)}\left[d_{G_{1}}\left(u_{1}\right)^{2}\right. \\
& u_{1} \in V\left(G_{1}\right), u_{2} \in V\left(Q\left(G_{1}\right)\right)-V\left(G_{1}\right) \\
& +d_{G_{2}}(v)^{2}+d_{Q\left(G_{1}\right)}\left(u_{2}\right)^{2}+2 d_{G_{1}}\left(u_{1}\right) d_{G_{2}}(v) \\
& \left.+2 d_{G_{1}}\left(u_{1}\right) d_{Q\left(G_{1}\right)}\left(u_{2}\right)+2 d_{G_{2}}(v) d_{Q\left(G_{1}\right)}\left(u_{2}\right)\right] \\
& =n_{2} F\left(G_{1}\right)+2 e_{1} M_{1}\left(G_{2}\right)+2 n_{2} F\left(G_{1}\right)+4 n_{2} M_{2}\left(G_{1}\right)+4 e_{2} M_{1}\left(G_{1}\right) \\
& +2 n_{2}\left(F\left(G_{1}\right)+2 M_{2}\left(G_{1}\right)\right)+8 e_{2} M_{1}\left(G_{1}\right) \\
& \therefore(2 a) \Rightarrow n_{2}\left(5 F\left(G_{1}\right)+8 M_{2}\left(G_{1}\right)\right)+2 e_{1} M_{1}\left(G_{2}\right)+12 e_{2} M_{1}\left(G_{1}\right) \\
& \begin{array}{c}
(2 b) \Rightarrow \sum_{v \in V\left(G_{2}\right)} \sum_{\substack{u_{1} u_{2} \in E\left(Q\left(G_{1}\right)\right) \\
u_{1}, u_{2} \in V\left(Q\left(G_{1}\right)\right)-V(G)}}\left[d_{Q\left(G_{1}\right)}\left(u_{1}\right)+d_{Q\left(G_{1}\right)}\left(u_{2}\right)\right]^{2} \\
\end{array}
\end{aligned}
$$




$$
=\sum_{v \in V\left(G_{2}\right)} \sum_{\substack{w_{i} w_{j} \\ w_{j} w_{k} \in E\left(G_{1}\right)}}\left[\left(d_{G_{1}}\left(w_{i}\right)+d_{G_{1}}\left(w_{j}\right)\right)+\left(d_{G_{1}}\left(w_{j}\right)+d_{G_{1}}\left(w_{k}\right)\right)\right]^{2} .
$$

$X_{i}$ and $X_{j}$ are vertices of $L\left(G_{1}\right)$, so we have

$$
\begin{aligned}
= & \sum_{X_{i} X_{j} \in E\left(L\left(G_{1}\right)\right)}\left[\left(d_{L\left(G_{1}\right)}\left(X_{i}\right)+d_{L\left(G_{1}\right)}\left(X_{j}\right)+4\right)^{2}\right] \\
= & \sum_{X_{i} X_{j} \in E\left(L\left(G_{1}\right)\right)}\left[d_{L\left(G_{1}\right)}\left(X_{i}\right)^{2}+d_{L\left(G_{1}\right)}\left(X_{j}\right)^{2}\right. \\
& \left.+16+2 d_{L\left(G_{1}\right)}\left(X_{i}\right) d_{L\left(G_{1}\right)}\left(X_{j}\right)+8 d_{L\left(G_{1}\right)}\left(X_{i}\right)+8 d_{L\left(G_{1}\right)}\left(X_{j}\right)\right] \\
= & H M\left(L\left(G_{1}\right)\right)+8 M_{1}\left(L\left(G_{1}\right)\right)+16\left(\frac{1}{2} M_{1}\left(G_{1}\right)-e_{1}\right) \\
(2)=(2 a)+(2 b)= & n_{2}\left(H M\left(L\left(G_{1}\right)\right)+8 M_{1}\left(L\left(G_{1}\right)\right)+8 M_{1}\left(G_{1}\right)-16 e_{1}\right)+5 F\left(G_{1}\right)+8 M_{1}\left(L\left(G_{1}\right)\right) \\
& \left.+8 M_{1}\left(G_{1}\right)+8 M_{2}\left(G_{1}\right)-16 e_{1}\right)+2 e_{1} M_{1}\left(G_{2}\right)+12 e_{2} M_{1}\left(G_{1}\right) .
\end{aligned}
$$

Therefore, (1) and (2) gives

$$
\begin{aligned}
H M\left(G_{1}+{ }_{Q} G_{2}\right)=n_{2}\left(H M\left(L\left(G_{1}\right)\right)+5 F\left(G_{1}\right)+8 M_{1}\left(L\left(G_{1}\right)\right)+8 M_{1}\left(G_{1}\right)\right. \\
\left.+8 M_{2}\left(G_{1}\right)-16 e_{1}\right)+10 e_{1} M_{1}\left(G_{2}\right)+16 e_{2} M_{1}\left(G_{1}\right)+n_{1} H M\left(G_{2}\right) .
\end{aligned}
$$

Theorem 3.4. Let $G_{1}$ and $G_{2}$ be two connected graphs. Then

$$
\begin{aligned}
H M\left(G_{1}+{ }_{T} G_{2}\right)=11 n_{2} F\left(G_{1}\right)+n_{1} F\left(G_{2}\right)+ & 24 e_{2} M_{1}\left(G_{1}\right) \\
& +12 e_{1} M_{1}\left(G_{2}\right)+6 n_{2} M_{2}\left(G_{1}\right) .
\end{aligned}
$$

Proof.

$$
\begin{aligned}
H M\left(G_{1}+{ }_{T} G_{2}\right)= & \sum_{\left(u_{1} v_{1}\right)\left(u_{2} v_{2}\right) \in E\left(G_{1}+{ }_{T} G_{2}\right)}\left[d\left(u_{1}, v_{1}\right)+d\left(u_{2}, v_{2}\right)\right]^{2} \\
= & \sum_{u \in V\left(G_{1}\right)} \sum_{v_{1}, v_{2} \in E\left(G_{2}\right)}\left[d\left(u, v_{1}\right)+d\left(u, v_{2}\right)\right]^{2} \\
& +\sum_{v \in V\left(G_{2}\right)} \sum_{u_{1}, u_{2} \in E\left(T\left(G_{1}\right)\right)}\left[d\left(u_{1}, v\right)+d\left(u_{2}, v\right)\right]^{2}
\end{aligned}
$$




$$
=(1)+(2) \text {. }
$$

By use of Theorem 3.2, we receive

$$
\begin{aligned}
& (1) \Rightarrow 16 e_{2} M_{1}\left(G_{1}\right)+16 e_{1} M_{1}\left(G_{2}\right)+n_{1} H M\left(G_{2}\right) \\
& (2) \Rightarrow \sum_{v \in V\left(G_{2}\right)} \sum_{u_{1} u_{2} \in E\left(T\left(G_{1}\right)\right)}\left[d\left(u_{1}, v\right)+d\left(u_{2}, v\right)\right]^{2} \\
& =\sum_{v \in V\left(G_{2}\right)} \sum_{u_{1} u_{2} \in E\left(T\left(G_{1}\right)\right)}\left[d\left(u_{1}, v\right)+d\left(u_{2}, v\right)\right]^{2} \\
& u_{1}, u_{2} \in V\left(G_{1}\right) \\
& +\sum_{v \in V\left(G_{2}\right)} \sum_{u_{1} u_{2} \in E\left(T\left(G_{1}\right)\right)}\left[d\left(u_{1}, v\right)+d\left(u_{2}, v\right)\right]^{2} \\
& u_{1} \in V\left(G_{1}\right), u_{2} \in V\left(T\left(G_{1}\right)\right)-V\left(G_{1}\right) \\
& +\sum_{v \in V\left(G_{2}\right)} \sum_{u_{1} u_{2} \in E\left(T\left(G_{1}\right)\right)}\left[d(u, v)+d\left(u_{2}, v\right)\right]^{2} \\
& u_{1}, u_{2} \in V\left(T\left(G_{1}\right)\right)-V\left(G_{1}\right) \\
& =(2 a)+(2 b)+(2 c) \text {. }
\end{aligned}
$$

By the use of Theorem 3.2, we receive

$$
\begin{aligned}
& (2 a)=4 e_{1} M_{1}\left(G_{2}\right)+4 n_{2} H M\left(G_{1}\right)+16 e_{2} M_{1}\left(G_{1}\right) \\
& (2 b)=\sum_{v \in V\left(G_{2}\right)} \sum_{u_{1}, u_{2} \in E\left(T\left(G_{1}\right)\right)}\left[d\left(u_{1}, v\right)+d\left(u_{2}, v\right)\right]^{2} \\
& u_{1} \in V\left(G_{1}\right), u_{2} \in V\left(T\left(G_{1}\right)\right)-V\left(G_{1}\right) \\
& =\sum_{v \in V\left(G_{2}\right)} \sum_{u_{1}, u_{2} \in E\left(T\left(G_{1}\right)\right)}\left[d_{T\left(G_{1}\right)}\left(u_{1}\right)\right. \\
& u_{1} \in V\left(G_{1}\right), u_{2} \in V\left(T\left(G_{1}\right)\right)-V\left(G_{1}\right) \\
& \left.+d_{G_{2}}(v)+d_{T\left(G_{1}\right)}\left(u_{2}\right)\right]^{2} \\
& =\sum_{v \in V\left(G_{2}\right)} \sum_{u_{1}, u_{2} \in E\left(T\left(G_{1}\right)\right)}\left[d_{R\left(G_{1}\right)}\left(u_{1}\right)^{2}\right. \\
& u_{1} \in V\left(G_{1}\right), u_{2} \in V\left(T\left(G_{1}\right)\right)-V\left(G_{1}\right) \\
& +d_{G_{2}}(v)^{2}+d_{Q\left(G_{1}\right)}\left(u_{2}\right)^{2}+2 d_{R\left(G_{1}\right)}\left(u_{1}\right) d_{G_{2}}(v) \\
& \left.+2 d_{R\left(G_{1}\right)}\left(u_{1}\right) d_{Q\left(G_{1}\right)}\left(u_{2}\right)+2 d_{G_{2}}(v) d_{Q\left(G_{1}\right)}\left(u_{2}\right)\right]
\end{aligned}
$$




$$
\begin{aligned}
& =\sum_{v \in V\left(G_{2}\right)} \sum_{u_{1}, u_{2} \in E\left(T\left(G_{1}\right)\right)}\left[4 d_{G_{1}}\left(u_{1}\right)^{2}\right. \\
& u_{1} \in V\left(G_{1}\right), u_{2} \in V\left(T\left(G_{1}\right)\right)-V\left(G_{1}\right) \\
& +d_{G_{2}}(v)^{2}+d_{Q\left(G_{1}\right)}\left(u_{2}\right)^{2}+4 d_{G_{1}}\left(u_{1}\right) d_{G_{2}}(v) \\
& \left.+2 d_{R\left(G_{1}\right)}\left(u_{1}\right) d_{Q\left(G_{1}\right)}\left(u_{2}\right)+2 d_{G_{2}}\left(v_{2}\right) d_{Q\left(G_{1}\right)}\left(u_{2}\right)\right] \\
& =4 n_{2} F\left(G_{1}\right)+M_{1}\left(G_{2}\right) 2 e_{1}+2 n_{2} H M\left(G_{1}\right)+4 M_{1}\left(G_{1}\right) 2 e_{2} \\
& +4 n_{2} H M\left(G_{1}\right)+2.2 e_{2}\left(2 M_{1}\left(G_{1}\right)\right) \\
& \therefore(2 b)=4 n_{2} F\left(G_{1}\right)+2 e_{1} M_{1}\left(G_{2}\right)+6 n_{2} H M\left(G_{1}\right)+16 e_{2} M_{1}\left(G_{1}\right) \\
& (2 c) \Rightarrow \sum_{v \in V\left(G_{2}\right)} \sum_{\substack{u_{1} u_{2} \in E\left(T\left(G_{1}\right)\right) \\
u_{1}, u_{2} \in V\left(T\left(G_{1}\right)\right)-V\left(G_{1}\right)}}\left[d\left(u_{1}, v\right)+d\left(u_{2}, v\right)\right]^{2} .
\end{aligned}
$$

By Theorem 3.3

$$
\begin{aligned}
(2 c)= & n_{2}\left(H M\left(L\left(G_{1}\right)\right)+8 M_{1}\left(L\left(G_{1}\right)\right)+8 M_{1}\left(G_{1}\right)-16 e_{1}\right) \\
\therefore(2)= & (2 a)+(2 b)+(2 c) \\
= & 6 e_{1} M_{1}\left(G_{2}\right)+32 e_{2} M_{1}\left(G_{1}\right)+n_{2}\left[H M\left(L\left(G_{1}\right)\right)+10 H M\left(G_{1}\right)\right. \\
& \left.+8 M_{1} L\left(G_{1}\right)+8 M_{1}\left(G_{1}\right)+4 F\left(G_{1}\right)-16 e_{1}\right] .
\end{aligned}
$$

Therefore, (1) and (2) gives

$$
\begin{aligned}
H M\left(G_{1}+{ }_{T} G_{2}\right) & =22 e_{1} M_{1}\left(G_{2}\right)+48 e_{2} M_{1}\left(G_{1}\right)+n_{1} H M\left(G_{2}\right)+n_{2}\left[H M\left(L\left(G_{1}\right)\right)\right. \\
& \left.+10 H M\left(G_{1}\right)+8 M_{1} L\left(G_{1}\right)+8 M_{1}\left(G_{1}\right)+4 F\left(G_{1}\right)-16 e_{1}\right] .
\end{aligned}
$$

\section{Conclusions}

New four sums (F-sums) of graphs introduced by Eliasi and Taeri are studied for The Wiener index. The Zagreb index have been found for the four operations on graphs by Hanyuan Deng et.al. recently.In this paper, Hyper-Zagreb index of four sums are studied for cartesian product. This can be extended to different topological indices and other products of graphs.

\section{References}

[1] V. Anandkumar, Radha Rajamani Iyer, Zagreb indices of the total graphs of graphs, Journal of Applied Sciences Research, 12, No. 3 (2016), 59-64. 
[2] B. Basavanagoud, S. Patil, A note on hyper-Zagreb index of graph operations, J. Appl. Math. Chem., 7, No. 1 (2016), 89-92.

[3] M. Azari, A. Iranmanesh, I. Gutman, Zagreb indices of bridge and chain graphs, MATCH Commun. Math. Comput. Chem., 70 (2013), 921-938.

[4] D.M. Cvetkovic, M. Doob, H. Sachs, Spectra of Graphs Theory and Application, Academic Press, New York, 1980.

[5] K.C. Das, K. Xu, I. Gutman, On Zagreb and Harary indices, MATCH Commun. Math. Comput. Chem., 70 (2013), 301-314.

[6] J. Devillers, A.T. Balaban (Eds.), Topological indices and related Descriptors in QSAR and QSPR, Amsterdam Netherlands, Girdon and Breach, pp.31 (1999), doi: $10.1080 / 10406630008028149$.

[7] M. Eliasi, B. Taeri, Four new sums of graphs and their Wiener indices, Discrete Appl. Math., 157 (2009), 794-803, doi: 10.1016/j.dam.2008.07.001.

[8] C.M.D. Fonseca, D. Stevanovic, Further properties of the second Zagreb index, MATCH Commun. Math. Comput. Chem., 72 (2014), 655-668.

[9] I. Gutman, K.C. Das, The first Zagreb index 30 years after, MATCH Commun. Math. Comput. Chem., 50 (2004), 83-92.

[10] I. Gutman, N. Trinajstic, Graph theory and molecular orbitals. Total $\pi$-electron energy of alternant hydrocarbons, Chem. Phy. Lett., 17 (1972), 535-538, doi: :10.1016/00092614(72)85099-1.

[11] Hanyuan Deng, D. Sarala, S.K. Ayyaswamy, S. Balachandran, The Zagreb indices of four operations on graphs, Appl. Math. Comput., 275 (2016), 422-431, doi: 10.1016/j.amc.2015.11.058.

[12] M.H. Khalifeh, H. Yousefi-Azari, A.R. Ashrafi, The first and second Zagreb indices of some graph operations, Discrete Appl. Math., 157 (2009), 804-811, doi: 10.1016/j.dam.2008.06.015.

[13] S. Li, H. Yang, Q. Zhao, Sharp bounds on Zagreb indices of cacti with $k$ pendent vertices, Filomat, 26 (2012), 1189-1200, doi: 10.2298/FIL1206189L.

[14] S. Li, M.J. Zhang, Sharp bounds for the Zagreb indices of bipartite graphs with a given diameter, Appl. Math. Lett., 24 (2011), 131-137, doi: 10.1016/j.aml.2010.08.032.

[15] S. Li, Q. Zhao, Sharp upper bounds on Zagreb indices of bicycle graphs with a given matching number, Math. Comput. Model., 54 (2011), 2869-2879, doi: 10.1016/j.mcm.2011.07.007.

[16] H. Lin, On segments, Vertices of degree two and the first Zagreb index of trees, MATCH Commun. Math. Comput. Chem., 72 (2014), 825-834.

[17] Muhammad Kamran Siddiqui, Muhammad Imran, Ali Ahmad, On Zagreb indices, Zagreb polynomials of some nanostar dendrimers, Appl. Math. Comput., 280 (2016), 132139, doi: 10.1016/j.amc.2016.01.041.

[18] U. Mary, P.S. Arockiaraj, A. James Albert, The first and second Zagreb indices of corona graphs, Int. J. Math. Archive, 5 (2014), 80-89.

[19] S. Nikolic, G. Kovacevic, A. Milicevic, N. Trinajstic, The Zagreb indices 30 years after, Croat. Chem., 60 (1996), 959-969. 
[20] D. Plavšić, S. Nikolić, N. Trinajstić, Z. Mihalić, On the Harary Index for the Characterization of chemical graphs, J. Math. Chem., 12 (1993), 235-250, doi: 10.1007/BF01164638.

[21] Wei Gao, Muhammad Kamran Jamil, Muhammad Reza Farahani, The Hyper-Zagreb index and some graph operations, J. Appl. Math. Comput. (2016), doi: :10.1007/s12190016-1008-9.

[22] G.H. Shirdel, H. Rezapour, A.M. Sayadi, Hyper-Zagreb index of graph operations, Iranian J. Math. Chem., 4, No. 2 (2013), doi: 213-220,10.22052/ijmc.2013.5294.

[23] H. Wiener, Structural determination of the paraffin boiling points, J. Amer. Chem. Soc., 69 (1947), 17-20, doi: 10.1021/ja01193a005.

[24] B. Zhou, I. Gutman, Further properties of Zagreb indices, MATCH Commun. Math. Comput. Chem., 54 (2005), 233-239. 